\title{
EVALUACIÓN DE ECOTIPOS LOCALES DE ALGODÓN DE COLOR (Gossypium Barbadense L. var.peruvianum EN LA ZONA DE TACNA.
}

\author{
Nelly Arévalo Solsol ${ }^{1}$ y Oscar Fernandez Cutire².
}

\section{RESUMEN}

En el centro experimental agrícola Los Pichones, se evaluó germoplasma de algodón de color, entre los años 1993 y 1994, con la finalidad de seleccionar ecotipos de algodón por rendimiento, determinar la variabilidad de las características de precocidad y capacidad productiva, y determinar el grado de asociación entre estas características. El material genético se obtuvo de una colección efectuada en diferentes localiáades de Tacna (La Yarada, Los Palos, Sama, y Locumba). Los resultados indican que se ha logrado seleccionar el genotipo LP 38.94 con buen rendimiento, precoz y buena arquitectura de planta. Las diferentes características evaluadas: altura de planta, longitud entre nudos, número de ramas de doble simpodio, número de bellotas cosechadas, peso de fibra, peso de semilla y rendimiento, presentaron alta variabilidad, lo cual indica la riqueza del material experimental que se podría aprovechar para futuros trabajos de mejoramiento genético. El rendimiento estuvo relacionado, positiva y significativamente, con el tamaño y peso de las bellotas cosechadas, y el peso y porcentaje de fibra

\section{INTRODUCCIÓN}

El algodón (Gossipium Barbadense var. peruvianum), llamado algodón de color, es una especie delaquese tiene poca investigación sobresu variabilidad genética, cuyo conocimiento se hace necesario para desarrollar variedades o ecotipos con buena calidad de fibra, buenos atributos agronómicos, rusticidad y resistencia a factores bióticos y abióticos, de modo que puedan ser aprovechados en la industria de los tejidos.

En la actualidad, la Facultad de Ciencias Agrícolas de la Universidad Nacional Jorge Basadre Grohmann(UNJBG), de Tacna, viene conduciendo un trabajo de investigación sobre evaluación de ecotipos locales de algodón de color. La colección, identificación

1. Magister en agronomía

2. Doctor en fruticultura y selección de muestras, nos permitirá obtener ecotipos de buena calidad de fibra y atributos agronómicos para futuros trabajos de mejoramiento.

El presente trabajo tiene como objetivos :

Seleccionar ecotipos de algodón de color de buen rendimiento y características agronómicas; determinar la variabilidad fenotípica, y establecer el grado de asociación entre las características evaluadas.

\section{MATERIALESYMETODOS UBICACIÓN}

El trabajo se instaló en el centro experimental agrícola Los Pichones (CEA III), de propiedad de la Facultad de Ciencias Agrícolas de la UNJBG , a 550 msnm, cuyas coordenadas geográficas son: 17.59، $38^{\prime \prime}$ de latitud sur y 70.14 '22" de longitud Oeste, durante la campaña de diciembre 1992 a junio 1994. 


\section{HISTORIADELCAMPOEXPERIMENTAL}

El campo experimental utilizado fue un terreno eriazo que anteriormente estuvo ocupado con maíz chalero, siendo los suelos deorigen aluvial con predominio de textura arenosa, típico de la zonas y regiones desérticas áridas y semiáridas

\section{MATERIALGENÉTICO}

Los materiales experimentales provienen de la colección de ecotipos de algodón de color (Gossipium Barbadense varperuvianum) tipo "aspero". Se obtuvo de unamezcla de una población que procededela colección de las zonas de la Yarada, Sama y los Palos.

\section{METODOLOGÍA}

Las submuestras se mezclaron para obtener una muestra homogénea y se sembraron a un distanciamiento de $1,5 \mathrm{~m}$ entre surcos y $0,9 \mathrm{~m}$ entre plantas, colocando dos semillas/golpe.

Se eligieron al azar 140 plantas competitivas que posteriormente fueron identificadas a 60 días después de la siembra y evaluadas posteriormente.

\section{CONDUCCIÓNDELEXPERIMENTO}

El cultivo se manejó en forma normal, aprovechando la gran rusticidad de estas plantas, tratando de obtener algodones orgánicos (cultivo sin ningún tipo de pesticidas y fertilizantes), encontrándose un alto número de controladores biológicos.

\section{CARACTERÍSJICASEVALUADAS}

Se evaluaron: el índice de inserción de primer simpodio, índice de inserción de la rama vegetativa, n. d $\epsilon$ doble simpodio en el tallo principal, longitud de los entrenudos, $n$. de ramas fruteras, altura de las plantas, peso de la bellota, porcentaje de fibra, índice de fibra, $n$. de bellotas/planta, rer -imiento de algodón en rama/ planta, acude.

\section{CRITERIOSDESELECCIÓNENCAMPO}

Además de tener un alto rendımiento, se tuvo también en cuenta los siguientes criterios: precocidad, resistencia a plagas y enfermedades, buen tamaño (peso de bellota),buen porcentaje de fibra, gran número de bellotas cosechadas.

\section{ANÁLISISESTADÍSTICO.}

En la población de estudio se determinó : La media, desviación estándar, el coeficiente de variabilidad y la correlación entre las características.

\begin{tabular}{|c|c|c|c|c|c|}
\hline Caracteres & & ngo & Media & sd & CV\% \\
\hline Altura de planta (cm) & 28 & -150 & 74,80 & 24,80 & 33,1 \\
\hline $\mathrm{N}^{\circ}$ ramas simpodiales & & -26 & 14,10 & 5,60 & 39,9 \\
\hline $\mathrm{N}^{\circ}$ dobles simpodios & 0 & -15 & 6,60 & 4,30 & 64,5 \\
\hline $\mathrm{N}^{\circ}$ bellotas formadas & & -55 & 18,60 & 11,30 & 60,5 \\
\hline Longitud de entrenudo $(\mathrm{cm})$ & 1,6 & $-14,7$ & 5,40 & 1,68 & 31,4 \\
\hline $\mathrm{N}^{\circ}$ de bellota cosech. & 0 & -33 & 7,40 & 7,87 & 106,9 \\
\hline Peso de bellota muestral & 0 & $-159,1$ & 30,52 & 33,16 & 108,7 \\
\hline Peso de bellota $(\mathrm{g})$ & 0 & $-6,40$ & 3,40 & 1,89 & 55,53 \\
\hline Peso fibra muestral (g) & 0 & $-54,8$ & 10,18 & 11,19 & 109,9 \\
\hline Peso semilla m.(g) & & $-104,2$ & 20,34 & 22,18 & 109,1 \\
\hline$\%$ de fibra & 23,3 & $-44,5$ & 33,25 & 4,72 & 14,2 \\
\hline$\%$ de semilla & 55,7 & $-76,6$ & 6,75 & 4,72 & 7,1 \\
\hline Indice de Fibra & 4,1 & $-11,1$ & 6,66 & 1,46 & 21,9 \\
\hline Acude & 1,7 & - 4,3 & 3,06 & 0,47 & 15,3 \\
\hline Rendimiento (g) & 2,8 & $-244,7$ & 47,12 & 51,75 & 109,9 \\
\hline
\end{tabular}

Los resultados del cuadro 1 indican que la altura de las plantas se encuentra en un rango de 28 a $150 \mathrm{~cm}$, tiene un promedio de $74,80 \pm 24,80$ y una variabilidad de $33,10 \%$.El número de ramas simpodiales posee un rango de 3 a I, una media de 14,10 $\pm 5,60$ y una variabilidad de $39,90 \%$. El número de doble simpodio muestra un rango de 0 al, una media de $6,60 \pm 4,30$ y una variabilidad de $64,50 \%$. El número de bellotas formadas posee un rango de 3 a 55 , una media de 18,60 11,30 y una variabilidad de 60,50.

La longitud de entre nudos presenta un rango 
entre 1,6 y $14,6 \mathrm{~cm}$, una media de $5,40 \pm 1,68$ y una variabilidad de $31,40 \%$. Basurto (1972) encontró valores entre $42 \mathrm{~mm}$ a $70 \mathrm{~mm}$ con un promedio general de 57,2 $\mathrm{mm}$.

El número de bellotas cosechadas posee un rango de 0 a 33 ,una media de $7,40 \pm 7,87$ y una variabilidad de 106,9.

El peso de bellota muestra un rango de 0 a 6,40 , una media de $3,40 \pm 1,89$ y un coeficiente de variabilidad de 55,53. Los datos son inferiores a lo encontrado por Basurto (1972), quien reporta valores de 3,62 a $5,57 \mathrm{gy}$ un promedio general de $4,59 \mathrm{~g}$. Ortega (1982) reporta valores de 5,31 a $4,3 \mathrm{~g}$ y un promedio general de 4,66 g.

El peso de fibra muestral posee un rango de 0 54,8 , una de $10,18 \pm 11,19$ y una variabilidad de $109,9 \%$. El peso de semilla muestral presenta un rango de 0 a 104,2 , una media de $20,34 \pm 22,18$ y una variabilidad de 109,1 .

El porcentaje de fibra posee un rango de 23,3a 44,5 , una media de $33,25 \pm 4,72$ y una variabilidad de $14,2 \%$, sin embargo, Basurto (1972) reporta valores de 36 a $45 \%$ para porcentaje de fibra y un promedio general de $38,06 \%$. En cambio, Chang (1978) en su estudio comparativo encuentra valores de 43,58 a 40,94 para porcentaje de fibra y un promedio general de $42,09 \%$; y Sánchez (1981), en su estudio comparativo de 49 linajes, encuentra valores de 41,60 a $39,47 \%$ para porcentaje de fibra, y un promedio general de $40,4 \%$.

El porcentaje de semilla tiene un rango de 4,1 a 11,1 , una media de $6,66 \pm 1,46$ y una variabilidad de $21,9 \%$.

Estos datos son inferiores a lo reportado por diferentes autores como Chang (1978), quien encontró valores de 8,22 a $9,16 \mathrm{~g}$ y un promedio general de 8,59 grs. Castro (1981) obtuvo valores de 8.85 a $7,41 \mathrm{~g}$ y un promedio general de $8,14 \mathrm{~g}$. Sánchez (1981) encontró valores de 8,61 a 7,30 g y un promedio general de 7,93 g.

El acude presenta un rango de 1,7 a 4,3 , una media de $3,06 \pm$ y una variabilidad de $15,3 \%$.

El rendimiento mostró un rango de 2,8 a 244,7 g, unamedia de 47,12 $\pm 51,75$ y una variabilidad de 109,9 $\%$. Todas estas características evaluadas tienen una alta variabilidad.

En el cuadro 2 se observa que existe una alta correlación positiva y significativa entre la altura de plantas y el número de ramas simpodiales y el número de dobles simpodios. El n. de ramas simpodiales con el 36 número de bellotas formadas y el número de dobles simpodios con el número de bellotas formadas. El número de bellotas cosechadas con el peso de bellota, peso de fibra, peso de semilla y el rendimiento.

El peso de bellota con el peso de fibra, peso de semilla, acude y el rendimiento. Estos resultados son similares a lo reportado por Straman (1930) y Basurto (1972), quienes afirman que el rendimiento se debe al número de bellotas y al peso por bellota. La meta debe ser producir un gran número de bellotas de alto peso, y que estas características estén correlacionadas:

El peso de semilla con el acude y el rendimiento; El peso de fibra con el peso semilla;

El peso de semilla con el acude y el rendimiento;

El porcentaje de fibra con el porcentaje de semilla, índice de fibra, acude y rendimiento. Grifee, Licon y Brannon (-1929-) señalan que el rendimiento de algodón en rama yel índice de fibra están correlacionados positivamente, pero hallaron valores significativos y no significativos ; $y$,

El porcentaje de semilla con el índice de fibra, acude y rendimiento. En las demás relaciones no existe correlaciones por tener correlaciones cercanas a cero.

Se han seleccionado 21 genotipos, sobresaliendo el genotipo LP.38.94 con un rendimiento de 244,7 gramos,seguido del genotipo LP.45.94 con 213,3 gramos. Los demás se encuentran en un rango de 70,3 y 244,7 .

Los genotipos que mostraron mayor precocidad fueron LP.123.94 y LP.200.94 con 15 y 12 bellotas formadas respectivamente y una altura relativamente alta de 111 y $117 \mathrm{~cm}$ cada uno. Estos resultados confirman loreportado porBasurto (1984), quienmenciona que la precocidad no está correlacionadacon la altura. Pueden haber plantas precoces y de buena altura.

\section{CONCLUSIONES}

\section{Capacidad Productiva}

1. El rendimiento de algodón en rama es la característica primordial, objeto de la selección hacia el logro de incre mento en los rendimientos unitarios en el algodonero.

2. El rendimiento de algodón en rama, depende del número y peso de bellotas cosechadas, los pesos de fibra y de semillas.

3. El peso de las bellotas depende del peșo de la fibra y del peso de las semillas.

4. Las semillas de mayor peso poseen mayor cantidad defibra.

\section{Precocidad}




\begin{tabular}{|c|c|c|c|c|c|c|c|c|c|c|c|c|c|c|}
\hline & $x 2$ & X3 & $X 4$ & $\times 5$ & $\mathrm{X} 6$ & $x 7$ & $x 8$ & X9 & $\mathrm{X} 10$ & $X 11$ & $\mathrm{X} 12$ & $X 13$ & $X 14$ & $\times 15$ \\
\hline$x_{1}$ & 0.51 & 0.61 & 0.57 & 0.01 & 0.18 & 0.19 & 0.19 & 0.18 & 0.03 & -0.03 & 0.05 & -0.03 & 0.21 & 0.02 \\
\hline$x_{2}$ & & 0.41 & 0.35 & 0.01 & 0.04 & 0.03 & 0.03 & 0.03 & -0.08 & -0.11 & -0.06 & -0.09 & 0.03 & -0.12 \\
\hline X3 & & & 0.59 & -0.01 & 0.05 & 0.07 & 0.09 & 0.07 & -0.03 & 0.07 & -0.01 & -0.06 & 0.07 & 0.01 \\
\hline $\mathrm{X} 4$ & & & & -0.05 & 0.19 & 0.19 & 0.28 & 0.19 & 0.05 & 0.02 & 0.03 & 0.02 & 0.20 & 0.10 \\
\hline X5 & & & & & -0.04 & 0.01 & -0.03 & 0.02 & -0.13 & -0.08 & $-0,13$ & -0.05 & -0.01 & -0.10 \\
\hline $\mathrm{X} 6$ & & & & & & 0.96 & 0.95 & 0.95 & 0.45 & 0.45 & 0.40 & 0.43 & 0.95 & 0.37 \\
\hline$\times 7$ & & & & & & & 0.99 & 0.99 & 0.44 & 0.45 & 0.39 & 0.42 & 0.98 & 0.46 \\
\hline$x 8$ & & & & & & & & 0.97 & 0.47 & 0.42 & 0.44 & 0.38 & 0.98 & 0.45 \\
\hline \begin{tabular}{|l|}
$\times 9$ \\
\end{tabular} & & & & & & & & & 0.42 & 0.45 & 0.37 & 0.44 & 0.98 & 0,46 \\
\hline$x_{10}$ & & & & & & & & & & 0.89 & 0.98 & 0.80 & 0.44 & 0.84 \\
\hline $\mathrm{X} 11$ & & & & & & & & & & & 0.82 & 0.97 & 0.44 & 0.88 \\
\hline$x 12$ & & & & & & & & & & & & 0.72 & 0.40 & 0.79 \\
\hline $\mathrm{X}_{13}$ & & & & & & & & & & & & & 0.40 & 0.83 \\
\hline$\overline{X 14}$ & & & & & & & & & & & & & & 0.45 \\
\hline
\end{tabular}

(1)

$X 1$. Altura de plantas expresado en $\mathrm{cm}$

$X 2 . N^{\circ}$ de ramas simpodiales

X. $N^{\circ}$ de doble simpodio

$X 4 . N^{\circ}$ de bellotas formadas

$X 5$. Longitud de entrenudo ex presado en $\mathrm{cm}$.

5. Las plantas que presentan mayor número de dobles simpodios y mayor número de ramas simpodiales tendrán, a su vez, mayor número de bellotas formadas.

6. Dentro de ciertos límites, el mayor tamaño de las plantas no excluye la posibilidad de que la planta sea precoz.

\section{Capacidad Productiva y Calidad de fibra.}

7. Los incrementos de rendimiento de algodón en rama son independientes de la calidad de la fibra.

8. En los algodones probados el rendimiento de algodón en rama y el índice de fibra están correlacionados.

\section{Precocidad y Capacidad Productiva}

9. Las plantas de mayor rendimiento presentan mayor tamaño y evolucionan más temprano en las yemas estraxilares en simpodios.

\section{BIBUOGRAFA}

BASURTO, L.A. 1972, Estudio de la precocidad, capacidad productiva y calidad de fibra y semilla en 145 linajes de algodón Tanguis. Tesis. Universidad Nacional Agraria La Molina. (UNALM), Lima-Perú.

- 1985, Heredabilidad y ganancia debido a la selección durante un ciclo de tres años de se lección en algodón Tanguis (Gossipiumbar abdense). Tesis UNALM, Lima-Perú.

CASTRO, J. 1981, Estudio comparativo de 36 linajes de algodón Tanguis (G. Barbadense). Tesis, UNALM, Lima-Perú.comparativo de 36 linajes de algodónTanguis. Tesis,UNALM,LimaPerú.

GRIFFE, F. LL. LICON Y BRANNON, s/f. Biometrical analy-
X11. Porcentaje de semilla

$X 12$. Indice de fibra

X13. Acude

$X 14$. Rendimiento en gramos

X15. Peso de bellota en gramos

10. Las plantas de menor precocidad preseritan mayor desarrollo de las bellotas con abundante fibra y mayor peso de las semillas.

11. La precocidad está asociada con el incremento en el número de bellotas formadas.

12. Bellotas con mayor peso de semillas provienen de plantas menos precoces.

13. Bellotas de mayor peso son logradas de plantas menos precoces y además poseerán alto porcentaje de fibra, alto porcentaje de semilla y alto índice de fibra.

14. Al incrementar los rendimientos se incrementan el número de bellotas.

\section{Genotipos Seleccionados.}

15. El genotipo LP. 38,94 mostró un rendimiento alto, buen tamaño de planta y una buena cantidad de bellotas formadas y precocidad.

sis of Upland cotton grown at Stillwater Okla homa Agrocultural and Mechanical College Agricul tural Experiment Station, Stillwater, Okla homa, Experment Bulletin 187.

ORTEGA C. J. 1982, Estudio comparativo de 30 cultivares Tanguis (G. barbadense) al punto devista de su valor agrícola e industrial. Tesis, UNALM, LimaPerú.

SANCHEZ, F. M. 1981, Estudio comparativo de 4 linajes de algodón Tanguis ( $G$. barbadense $L$,). Tesis, UNALM, Lima-Perú.

STROMAN, N. G. 1930, Biometrical relationship of cetain characters in Upland cotton. Journal ofAmerican Society of Agronomy. 22 (4). 